\title{
ARTICLE
}

Received 2 Oct 2013 | Accepted 17 Dec 2013 | Published 23 Jan $2014 \quad$ DOl: 10.1038/ncomms4129

\section{Reactive oxygen species mediate pollen tube rupture to release sperm for fertilization in Arabidopsis}

\author{
Qiaohong Duan ${ }^{1, \star}$, Daniel Kita ${ }^{1,2, \star, \dagger}$, Eric A. Johnson ${ }^{1,2}$, Mini Aggarwal ${ }^{1,3}$, Laura Gates ${ }^{1, \dagger}$, \\ Hen-Ming $\mathrm{Wu}^{1,2} \&$ Alice Y. Cheung ${ }^{1,2,3}$
}

In flowering plants, sperm are transported inside pollen tubes to the female gametophyte for fertilization. The female gametophyte induces rupture of the penetrating pollen tube, resulting in sperm release and rendering them available for fertilization. Here we utilize the Arabidopsis FERONIA (FER) receptor kinase mutants, whose female gametophytes fail to induce pollen tube rupture, to decipher the molecular mechanism of this critical male-female interactive step. We show that FER controls the production of high levels of reactive oxygen species at the entrance to the female gametophyte to induce pollen tube rupture and sperm release. Pollen tube growth assays in vitro and in the pistil demonstrate that hydroxyl free radicals are likely the most reactive oxygen molecules, and they induce pollen tube rupture in a $\mathrm{Ca}^{2+}$. dependent process involving $\mathrm{Ca}^{2}+$ channel activation. Our results provide evidence for a RHO GTPase-based signalling mechanism to mediate sperm release for fertilization in plants.

\footnotetext{
${ }^{1}$ Department of Biochemistry and Molecular Biology, University of Massachusetts, 710N. Pleasant Street, Lederle Graduate Research Tower, Amherst, Massachusetts 01003, USA. ${ }^{2}$ Molecular Cell Biology Program, University of Massachusetts, 710N. Pleasant Street, Lederle Graduate Research Tower, Amherst, Massachusetts 01003, USA. ${ }^{3}$ Plant Biology Graduate Program, University of Massachusetts, 710N. Pleasant Street, Lederle Graduate Research Tower, Amherst, Massachusetts 01003, USA. * These authors contributed equally to this work. †Present addresses: Department of Vascular Biology, University of Connecticut Health Center, Farmington, Connecticut 06030, USA (D.K.); Department of Biology, University of California, La Jolla, California 92093, USA (L.G.). Correspondence and requests for materials should be addressed to A.Y.C. (email: acheung@biochem.umass.edu).
} 
U nlike fertilization in animal where flagellated sperm swim to fuse with the oocyte, sperm cells in flowering plants are immotile. They are borne inside the pollen tube, the male gametophyte, and rely on its polarized growth inside the female organ, pistil, for transportation and delivery to the female gametophyte located inside an ovule for fertilization (Fig. 1a $)^{1-3}$. The egg cell and a pair of synergid cells are located at one end of the female gametophyte, close to the ovular aperture called micropyle. Pollen tube growth in the pistil is highly regulated and fertilization is achieved only after a series of interactive events between the penetrating pollen tube and specific pistil tissues ${ }^{4-6}$ and cells ${ }^{7,8}$. Guided by female cues ${ }^{7,8}$, the pollen tube enters the ovule via the micropyle. The filiform apparatus is located at the entrance of the female gametophyte. It is a thickened cell wall region crisscrossed by an abundance of cell membrane protrusions from the synergids ${ }^{9,10}$, the pair of cells flanking the egg (Fig. 1a). The pollen tube invades one of the synergids whereupon it ruptures, ejecting sperm and rendering them available for fertilization 3,11 . Late-arriving pollen tubes are repelled from entering an already penetrated ovule.

Mutant analyses showed that the female gametophyte plays a determining role in inducing sperm release and preventing latearriving tubes from entering an already penetrated ovule ${ }^{12-17}$ while the male gametophyte is also actively involved to ensure reproductive success ${ }^{18-21}$. Among these mutants, ovules in lossof-function mutants in the Arabidopsis FERONIA/SIRENE receptor kinase (FER/SRN, FER is used from here on except when referring to the srn mutant) show the strongest dual phenotype of failing to induce pollen tube rupture and preventing multiple tube entrance. As a consequence, fer ovules exhibit a readily notable phenotype of pollen tube pile up (Supplementary Fig. 1a) due to overgrowth of one or more pollen tubes inside the female gametophyte $\mathrm{e}^{12,13}$.

FER is a member of the Arabidopsis THESEUS1/FER receptorlike kinase (RLK) family ${ }^{22}$; the functions of THESEUS1, which regulates growth in cellulose-deficient seedlings, and FER are the first in the family to be elucidated ${ }^{23,24}$. FER is broadly expressed $^{24,25}$, except in the pollen ${ }^{26}$, where several related RLKs are expressed ${ }^{26-28}$. Two of these pollen-specific RLKs, ANXUR1 and ANXUR2, function redundantly to ensure pollen tube integrity $^{27,28}$. Double anxur1/2 mutants show precocious pollen tube rupture in the pistil, never reaching the ovules for fertilization. FER is also important for overall growth and development, regulating cell growth, hormone-signalled responses $^{25,29-31}$, and facilitates pathogen invasion ${ }^{16}$. We showed previously ${ }^{25}$ that FER interacts with ROPGEFs ${ }^{32}$, guanine nucleotide exchange factors that activate RAC/ROP GTPases (plant RHOs) and major molecular switches that mediate myriad signal-response systems ${ }^{33-35}$. We showed that FER functions as a cell surface regulator in a FER-ROPGEF-RAC/ ROP complex to stimulate nicotinamide adenine dinucleotide phosphate (NADPH) oxidase-produced reactive oxygen species (ROS) in roots and mediate polarized root hair growth ${ }^{25}$. Since FER interacts with multiple ROPGEFs, which are known to interact with distinct classes of effectors ${ }^{36,37}$, whether FER uses the same or other RAC/ROP-regulated pathways to mediate its various functional roles has to be established on a case by case basis. On the other hand, despite the accumulating knowledge on the genetic control over male-female gametophyte interactions ${ }^{3,11}$, insights pertaining to the mechanisms that underlie these processes remain sparse due to the difficulty to access and manipulate the microenvironment for the malefemale encounter. Thus far, only a female gametophyte produced defensin-like protein, ZmES4, from maize has been shown to burst pollen tubes, acting preferentially on maize pollen tubes ${ }^{17}$. Therefore, discovery of a FER to ROS signalling pathway a

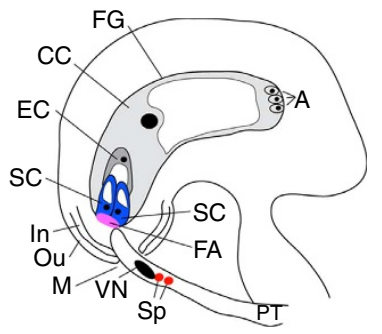

b
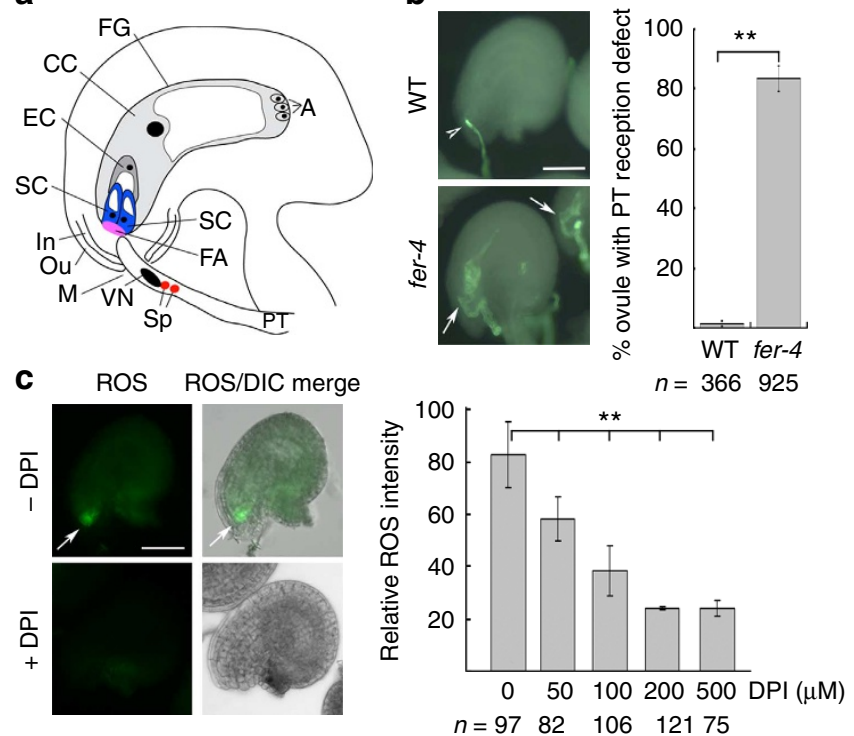

d
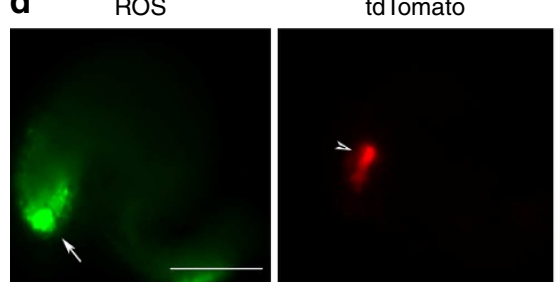

ROS/tdTomato/DIC

e
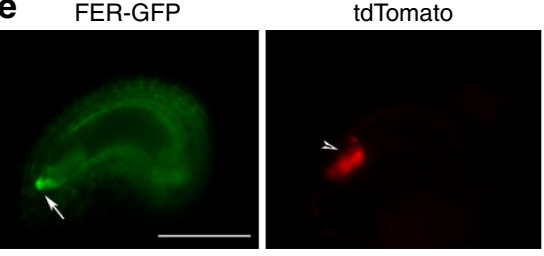

FER-GFP/tdTomato

Figure 1 | FER mediates a ROS maximum at the filiform apparatus/ synergid cell region. (a) An ovule sketch showing a penetrating pollen tube. A, antipodal cells; FG, female gametohyte; CC, central cell; EC, egg cell; SC, synergid cell; FA, filiform apparatus; In, Oi, inner and outer integuments; $M$, micropyle; $\mathrm{PT}$, pollen tube; $\mathrm{VN}$, pollen tube vegetative nucleus; Sp, sperm cell. (b) Aniline blue-stained WT and fer-4 ovules (left) and quantification of pollen tube (PT)-ovule interaction defects (right). Arrowhead, a single, ruptured tube; arrows, multiple overgrown (reflecting non-rupture) tubes appearing as thick bundles. Data = average \pm s.e.m. from at least triplicate samples (each with at least three pistils) from a representative analysis; $n=$ total number of ovules examined. ${ }^{\star \star} P \leq 0.01$ by Student's t-test. (c) $\mathrm{H}_{2}$ DCF-DA staining to show ovular ROS (left panel) and dose-dependent DPI inhibition (right panel). For comparative ROS analysis, a region of interest (ROI) with identical areas at the synergid cell/ filiform apparatus region (Supplementary Fig. 2) from the ovules compared was quantified by Image J. Data in histogram $=$ average \pm s.d. of ovules from at least three pistils ( $n=$ number of ovules examined) from a representative of three independent analyses. (d,e) Co-imaging of the discharged pollen tube cytoplasm (red, arrowheads) and ROS (green, arrows) in a wild-type ovule (d) and of pollen tube cytoplasm (red, arrowheads) and with FER-GFP (green, arrows) in a pFER::FER-GFP transformed ovule (e).

operating in roots ${ }^{25}$ provided a valuable portal to explore how the female gametophyte controls the fate of the invading male gametophyte. Here we show that FER induces a high ROS environment at the entrance of the female gametophyte and that 
it is required for inducing pollen tube rupture. In particular, we show that inhibiting female gametophytic ROS production phenocopies the fer ovule phenotype of pollen tube overgrowth and provide evidence for ROS-induced pollen tube rupture as a $\mathrm{Ca}^{2+}$-dependent process in vitro and in the pistil. Our results establish a model whereby FER interacts with the RAC/ROP molecular switch and acts via a NADPH oxidase-dependent signalling pathway to induce sperm release and enable fertilization in flowering plants.

\section{Results}

FER mediates high ROS levels at the filiform apparatus area. As the FER-regulated reproductive phenotype was originally described in heterozygous $s r n^{12}$ and fer $^{13}$ mutants and our study would be carried out in the homozygous T-DNA induced fer-4 null mutant ${ }^{25}$, we first ascertained that $f e r-4$ has a reproductive phenotype indistingusishable from those reported in srn and fer. We established that pollen tubes failed to rupture in about $80 \%$ of the fer- 4 ovules and that about half of these were also penetrated by multiple pollen tubes (Fig. 1b; Supplementary Fig. 1a,b), levels that approximately doubled those reported in heterozygous srnand fer-mutant pistils. We also confirmed that reduced fertility in fer-4 was female dependent (Supplementary Fig. 1c,d), just as previously reported for the heterozygous srn and fer.

To examine whether female gametophyte-expressed $\mathrm{FER}^{24}$ (Supplementary Fig. 2) regulates ROS production, wild-type ovules were stained for ROS in the absence and presence of diphenyl iodonium chloride (DPI), an inhibitor commonly used to assess cell membrane NADPH oxidase-dependent ROS synthesis (Supplementary Fig. 3) 25,38,39. Results showed a DPIsensitive ROS maximum at the filiform apparatus/synergid cell region (Fig. 1c; Supplementary Fig. 2), where FER is prominently located $^{24}$ (Supplementary Fig. 2). We also co-imaged red fluorescent cytoplasm discharged from ruptured tdTomatoexpressing pollen tubes in the synergid cell with ROS in wildtype ovules (Fig. 1d) or with FER-GFP in ovules from FER promoter (pFER)::FER-GFP transformed plants ${ }^{25}$ (Fig. 1e). Results showed a close spatial relationship between FER and ROS at the entrance to the female gametophyte, just external to the red fluorescence-labelled pollen tube cytoplasm.

We then examined the ROS status and FER expression during floral development ${ }^{40,41}$ (Supplementary Fig. 4a) and compared the ROS status between wild type and fer-mutant ovules. pFERexpressed FER-GFP and ROS both showed a developmentally regulated accumulation pattern in wild-type ovules, peaking during floral stages 13-15 (Fig. 2a) when ovules were at their prime receptivity for pollen tubes ${ }^{40,41}$ (See Supplementary Fig. 4a), and dropping by stage 16 when fertilization was complete. On the other hand, ROS level remained low in fer-4 throughout development (Fig. 2b,c; Supplementary Fig. 4b,c). The female gametophytic ROS status in $s r n$ showed a similar developmental regulation profile (Fig. 2d), further ascertaining that loss of a high ROS environment at the entrance to the female gametopyte has resulted from loss of FER function. Taken together, these observations are consistent with FER mediating a NADPH oxidase-dependent ROS maximum at the filiform apparatus/synergid cell region of the female gametophyte and that ROS potentially play a role in the FER-regulated reproductive processes.

Female gametophytic ROS are required for pollen tube rupture. Lacking genetic suppressors for fer mutants that were originally reported in 2003 (refs 12,13), and without a loss-offunction NADPH oxidase mutant that does not severely impair growth and development rendering precise interpretation of reproductive anomalies difficult, we devised a pistil-feeding assay to manipulate the pistillate environment (Fig. 3a; Supplementary Fig. 5a,b) to determine whether ROS indeed underlie FER-regulated pollen tube-ovule interactions. Feeding wild-type pistils with DPI effectively suppressed ovule ROS (Supplementary Fig. $5 c, d)$ and pollen tube rupture, resulting in their overgrowth (Fig. 3b,c, white bars). Similarly, scavenging superoxide and hydroxyl radical (see Supplementary Fig. 3) reduced ovule ROS and prevented pollen tube rupture (Fig. 3d,e; Supplementary Fig. 5e). Together, these observations provide strong support for ROS being required for pollen tube rupture in the female gametophyte. On the other hand and unlike the dual phenotype of pollen tube overgrowth and supernumerary pollen tube entrance in fer ovules, obliterating female gametophytic ROS and preventing pollen tube rupture had no impact on the wild-type ovule ability to prevent multiple tube entrance (Fig. 3b,c, grey bars). These results show that although intimately linked, the two FER-regulated pollen tube-ovule interactive processes can be uncoupled.

ROS induce pollen tube rupture in vitro. To further decipher the mechanism behind the ROS-induced pollen tube rupture process, we used the semi-in vivo pollen tube growth system ${ }^{4,42}$ to explore how pollen tubes would respond to applied ROS. Arabidopsis pollen tubes emerging from pollinated pistils cultured on standard growth medium showed robust growth for at least 4 $6 \mathrm{~h}$ (Fig. 4a). To generate a high ROS condition, we added xanthine/xanthine oxidase $(\mathrm{X} / \mathrm{XO})$ to convert molecular oxygen to superoxide, or applied $\mathrm{H}_{2} \mathrm{O}_{2}$ directly, or used $\mathrm{FeSO}_{4}$ to generate hydroxyl radicals via the Fenton reaction $38,43,44$ (Supplementary Fig. 3). All of these applications caused an abrupt and explosive discharge of pollen tube cytoplasm and sperm cells (Fig. 4a-c; Supplementary Fig. 6a-d; Supplementary Movies 1,2). Hydroxyl radical scavengers effectively suppressed rupture induced by all three reagents (Fig. 4d-f; Supplementary Fig. 6e-g). These observations imply that the $\mathrm{X} / \mathrm{XO}$-generated superoxide and the directly added $\mathrm{H}_{2} \mathrm{O}_{2}$ had predominantly acted via their conversion into hydroxyl radicals. Taken together, with observations from pistil-feeding assays (Fig. 3; Supplementary Fig. 5), these results also implicate hydroxyl radicals as the most prevalent active oxygen species that induce pollen tube rupture in the female gametophyte.

ROS-induced pollen tube rupture is $\mathrm{Ca}^{2+}$-dependent. To further probe the underlying process that lead to pollen tube rupture, we explored what potential targets ROS might use to trigger this response. Pollen tube growth is highly dependent on calcium $^{45}$ and ROS signalling is intimately linked to that of ion channel activation for example, that of $\mathrm{Ca}^{2+}$ and $\mathrm{K}^{+}$ (refs 39,43,44). Indeed, when pollen tubes were treated with ROS under different $\mathrm{Ca}^{2}+$ conditions, tube rupture was suppressed in response to declining $\left[\mathrm{Ca}^{2+}\right]$ in the medium (Fig. 5a). Application of $\mathrm{Ca}^{2+}$ channel blockers significantly reduced ROS-induced pollen tube rupture (Fig. 5b,c; Supplementary Fig. $7 \mathrm{a}, \mathrm{b})$, whereas addition of even high concentrations of the $\mathrm{K}^{+}$channel inhibitor $\mathrm{CsCl}$ did not (Fig. 5b; Supplementary Fig. 7c,d). Elongating pollen tubes are known to maintain a steep, tip-focused $\left[\mathrm{Ca}^{2+}\right]$ gradient ${ }^{45}$. Using Arabidopsis pollen tubes expressing a $\mathrm{Ca}^{2}+$ reporter ${ }^{46}$, we observed abrupt increases in $\mathrm{Ca}^{2}+$ in the distal cytoplasm within 1-2 min of ROS application (Fig. 5c; Supplementary Movies 3,4) and immediately preceding pollen tube rupture. $\mathrm{Ca}^{2}+$ channel inhibitors effectively blocked $\mathrm{Ca}^{2+}$ influx and inhibited rupture for at least half an hour in the presence of ROS, even when the apical $\mathrm{Ca}^{2+}$ gradient was dissipated and growth became depolarized (Fig. 5d). In the 


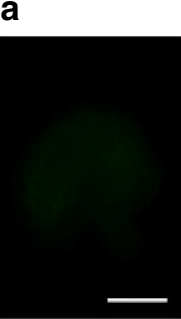

Stage 12

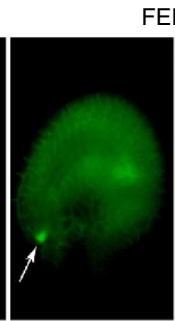

Stage 13
FER-GFP

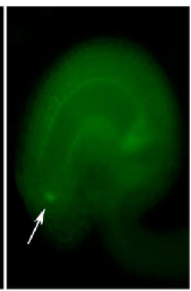

Stage 15

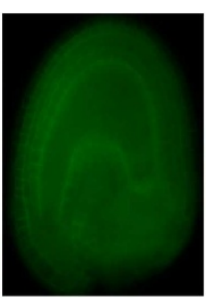

Stage 16

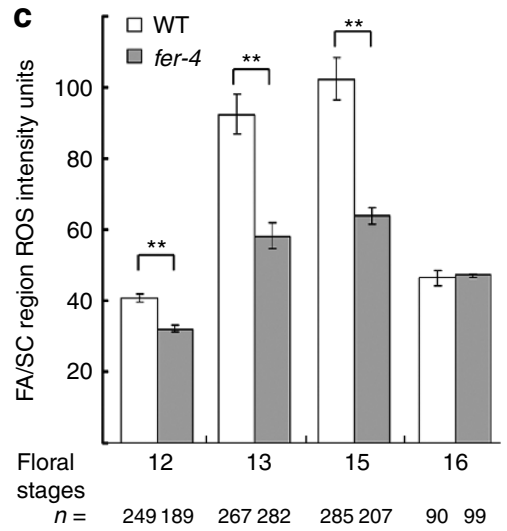

b
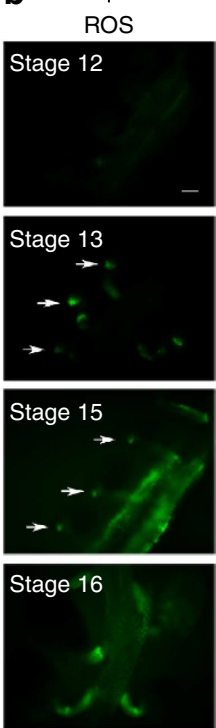

WT
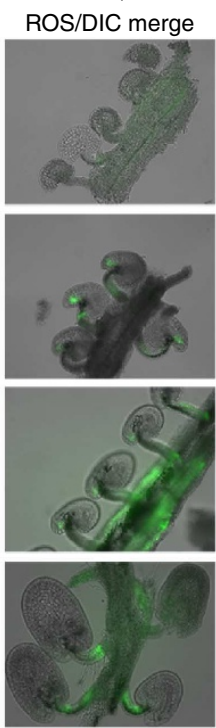

d

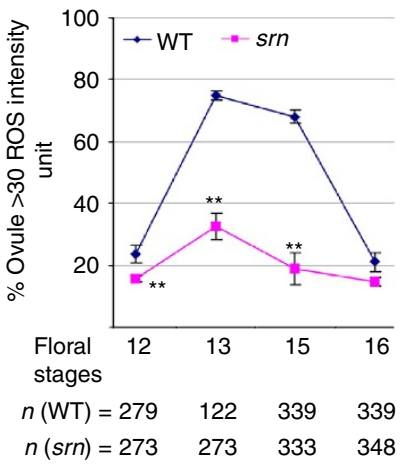

Figure 2 | FER mediates a developmentally regulated ROS maximum at the entrance to the female gametophyte. (a) $p F E R:: F E R-G F P$ expression in floral developmental stages 12-16 (refs 40,41). Arrows indicate filiform apparatus/synergid cell region. (b) $\mathrm{H}_{2} \mathrm{DCF}-\mathrm{DA}$-stained wild-type and fer-4 ovular ROS. Arrows indicate micropylar regions. (c,d) Quantitative analysis of $\mathrm{H}_{2}$ DCF-DA-detected female gametophytic ROS in wild-type and fer-4 (c) and in wild-type and srn (d) ovules. (c) compares average filiform apparatus/synergid cell (FA/SC) region ROS intensity units; (d) compares \% of ovules with ROS intensity above the baseline threshold set at 30 ROS intensity units. Both methods, and a third, comparing the ratio between FA/SC ROS and chalazal end ROS (around the antipodal cells), and comparisons using a second ROS-stain, HPF 38,69 (Supplementary Fig. 4b,c), yielded comparable results. Quantitative data $(\mathbf{c}, \mathbf{d})$ are averages \pm s.e.m. of triplicate samplings (each with at least three pistils) from an analysis representative of three independent experiments; ${ }^{\star \star}$, significant differences, $P<0.01$ by Student's $t$-test. $n=$ total number of ovules examined. Scale bar, $50 \mu$ m.

ovules, progressively reducing $\left[\mathrm{Ca}^{2+}\right]$ in the pistil-feeding medium or adding EGTA to deplete endogenous $\mathrm{Ca}^{2+}$ in excised pistils inhibited pollen tube rupture, inducing overgrowth (Fig. 6). Taken together, these observations show that ROSinduced pollen tube rupture in vitro is dependent on $\mathrm{Ca}^{2+}$ and $\mathrm{Ca}^{2+}$ channel activation. They are also consistent with $\mathrm{Ca}^{2+}$ being important for ROS-induced pollen tube rupture in the female gametophyte.

LORELEI also regulates female gametophytic ROS. To ascertain that a high ROS condition at the entrance to the female gametophyte is indeed a predominant condition for mediating pollen tube rupture in the female gametophye, we examined the ROS status in the ovules of a fer-like mutant lacking the glycosylphosphatidylinositol-anchored protein $\mathrm{LRE}^{14,15}$. We confirmed that pollen tube overgrowth in lre-5 (ref. 15) approximated the level observed in fer-4 and occurred at levels significantly higher than in wild-type ovules, similar to previously reported
(Supplementary Fig. 8). ROS staining showed that lre-5 ovules also showed significantly reduced levels of ROS around the filiform apparatus/synergid cell region relative to wild-type ovules (Fig. 7). That two molecules commonly mediate a high ROS environment in the female gametophyte and loss of their functions induces similar pollen tube overgrowth defects provide strong support for the high ROS region at female gametopyte entrance as critically linked to its ability to induce rupture of a penetrating pollen tube.

The similar ROS and pollen tube overgrowth phenotypes in fer and lre female gametophytes led to our considering that FER and LRE might act in a common pathway. We showed previously that FER interacts with ROPGEFs ${ }^{25,31}$ and coexists with RAC/ROP GTPases in a tripartite signalling complex ${ }^{25}$. Using the same assay, we observed that LRE, like FER, was pulled down by ROP2 (Fig. 7c), thus implicating LRE as another component in the FER-RAC/ROP signalling complex working in concert with FER to impact female gametophytic ROS production. 
a

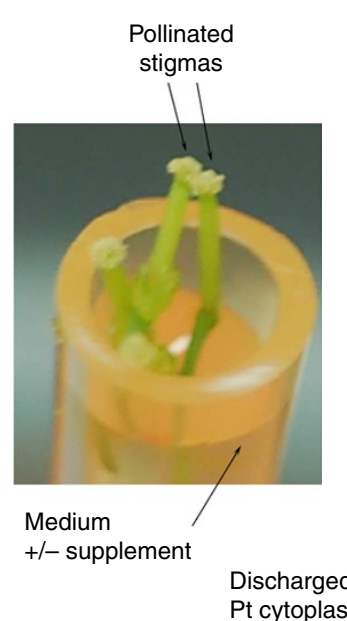

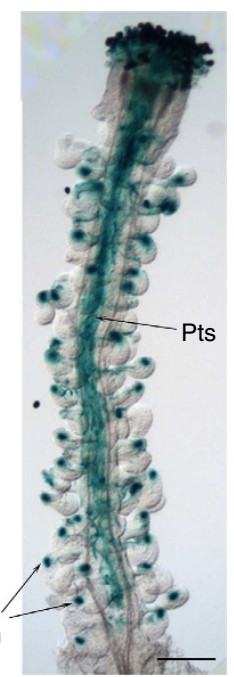

d b

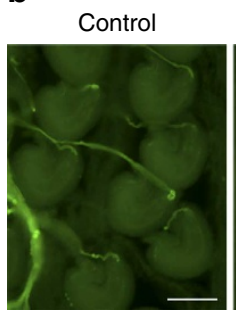

Tiron

$(10 \mathrm{mM})$

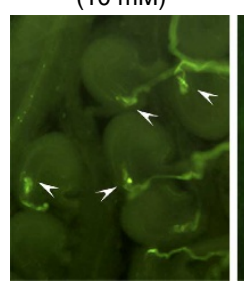

e
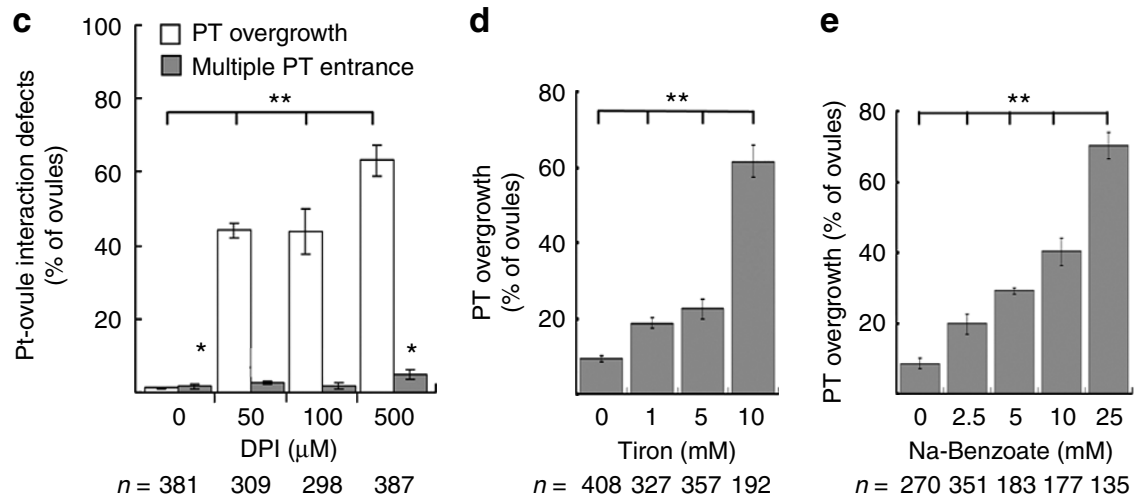

Figure 3 | ROS induce pollen tube rupture in the female gametophytes. (a) Pistil-feeding assay. Excised wild-type pistils maintained in a reservoir of feeding medium and a single wild-type pistil pollinated by GUS-expressing pollen highlighting ovule targeting and cytoplasmic discharge (blue dots) in the female gametophytes. Supplementary Figure 5 a shows an aniline blue-stained pollinated pistil after growth in the pistil-feeding assay, also confirming that normal pollen tube growth and ovule targeting were not affected. When fer- 4 pistils were maintained in the pistil-feeding assays, they retained pollen tube-ovule interaction defects, harbouring comparable levels of pollen tube overgrowth as seen in pistils pollinated in planta (Supplementary Fig. 5b), confirming that the FER-regulated process was not affected in these assays. (b-e) Pollen tube rupture and entrance property in ovules from mock-treated (control) wild-type pistils and those fed with DPI, tiron and Na-benzoate. DPI inhibition is commonly used as a reflection of NADPH oxidasedependent ROS synthesis, tiron and Na-benzoate are commonly used ROS scavengers ${ }^{67}$ (Supplementary Fig. 3). Arrowheads in (b) indicate overgrown single-pollen tubes. ROS levels were reduced to almost non-detectable level in these treated ovules (Supplementary Fig. $5 \mathrm{c}$,d). CuCl 2 , another ROS scavenger (Supplementary Fig. 3), also reduced ovular ROS and induced pollen tube overgrowth (Supplementary Fig. 5d,e). Data =average \pm s.e.m. from at least triplicate samples (each with at least three pistils) representative of three independent experiments; $n=$ total number of ovules examined. ${ }^{\star} P \leq 0.01 ;{ }^{\star} P \leq 0.05$ as determined by Student's $t$-tests. Scale bars, $250 \mu \mathrm{m}$ (a); $100 \mu \mathrm{m}$ (b).

\section{Discussion}

The discovery that FER-regulated ROS underlie reproductive success in Arabidopsis reveals a novel functional role for these ubiquitous regulatory molecules and provides mechanistic insight into a male and female interactive process that is crucial for flowering plant reproduction. ROS function in diverse ways ${ }^{47,48}$. For pollen tubes, ROS are required for their polarized growth ${ }^{49,50}$ but they also mediate death as shown here (Figs 3-6) and in selfincompatibility factor-treated pollen tubes ${ }^{51}$, suggesting different ROS-triggered processes could be involved. Plant cell growth requires constant balancing between cell wall extensibility to allow expansion and rigidity to provide integrity ${ }^{52}$ and ROS are important for maintaining a balance between these two conditions $38,39,47$. A regulated ROS environment, for example via signalling through $\mathrm{RAC} / \mathrm{ROPs}^{25,53}$, is important for cell growth such as in pollen tubes and root hairs. High levels of exogenously encountered ROS in the female gametophyte could over sever polysaccharides ${ }^{54}$ and weakens the pollen tube wall, resulting in tube rupture, similar to enzyme treatment that disrupts cell wall properties ${ }^{55}$.

The characteristics of ROS-induced pollen tube rupture described here provide insight on what might underlie the process. The $\mathrm{Ca}^{2+}$-dependent process in vitro and in the pistil suggests that $\mathrm{Ca}^{2+}$-signalled downstream events in the pollen tube could be involved. However, the almost instantaneous response upon exposure to ROS in vitro and penetration of the synergid cell in vivo would suggest that few pollen tube cytoplasmic signalling events are needed. The explosive nature of ROS-induced cytoplasmic and sperm discharge immediately following $\mathrm{Ca}^{2+}$ channel opening (Figs 4a,b and 5c; Supplementary Movies 1-4) suggest possibly that a disrupted force equilibrium between turgor and cell wall strength underlies tube bursting. That medium osmolarity can modulate this process under normal and ROS-challenged growth conditions (Supplementary Fig. 7e,f) are consistent with this proposition. Although spatial constraint could not have been a factor in the ROS-induced cata- 

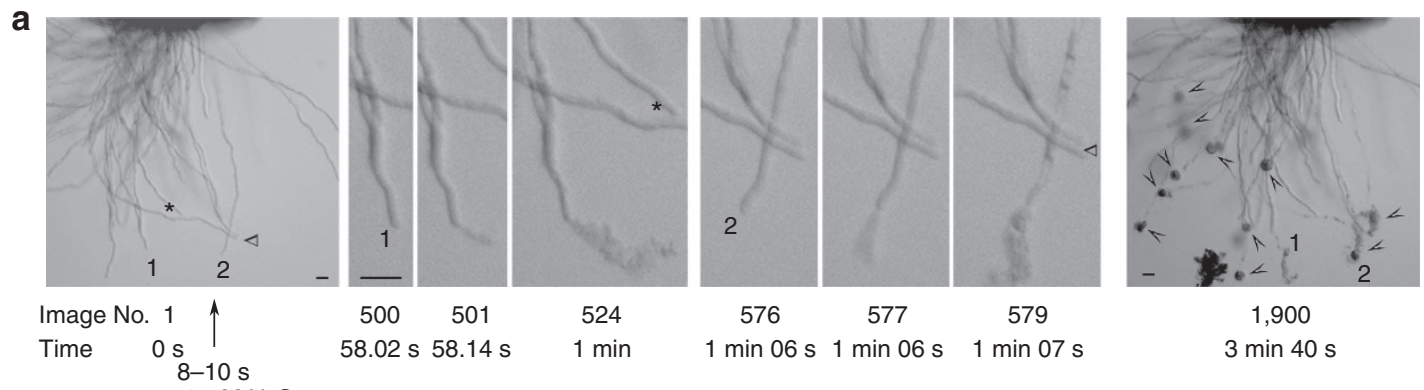

$+1 \mathrm{mM} \mathrm{H}_{2} \mathrm{O}_{2}$

b

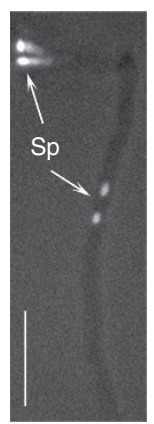

c

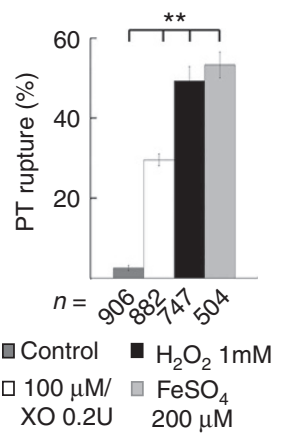

d $\quad 100 \mu \mathrm{MX}$ $0.2 \cup \times O$

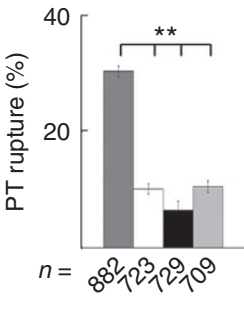

Key for $d-f$ e

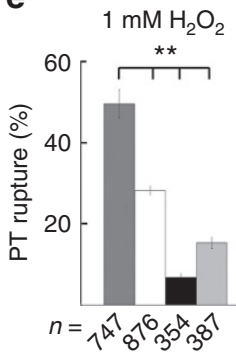

$\square$ Control

$\square$ Adenine $100 \mu \mathrm{M}$
1,900

$3 \mathrm{~min} 40 \mathrm{~s}$

Figure 4 | ROS induce pollen tube rupture in vitro. (a) $\mathrm{H}_{2} \mathrm{O}_{2}$-induced tube rupture. The entire sample immediately prior to application of $\mathrm{ROS}(\mathrm{O} \mathrm{s}$ ) and when a majority of the pollen tubes has ruptured ( $3 \mathrm{~m} 40 \mathrm{~s}$ ) (arrowheads point to cytoplasmic discharges) is shown in full. Magnified views of the first two rupturing pollen tubes $(1,2)$ are shown in between. ${ }^{\star}$ and $\Delta$ are reference points for tubes 1,2 . Supplementary Movie 1 shows the entire time series in real time. (b) $\mathrm{FeSO}_{4}$ induced RFP-labelled sperm cell (Sp) discharge. The image was captured in a single 250 ms exposure. Supplementary Movie 2 shows a time series of ROS-induced sperm release. Scale bars $(\mathbf{a}, \mathbf{b}), 50 \mu \mathrm{m}$. (c) Quantification of superoxide (generated by X/XO), $\mathrm{H}_{2} \mathrm{O}_{2}$ and hydroxyl free radicals (generated by $\mathrm{FeSO}_{4}$-mediated Fenton reaction) induced pollen tube rupture. (d-f) Hydroxyl free radical scavengers suppressed ROS-induced tube rupture. Data = average \pm s.e.m. from at least triplicate from an analysis representative of three independent experiments; $n=$ total number of pollen tubes examined. ${ }^{\star \star} P \leq 0.01$ by Student's $t$-tests.

strophic failure in pollen tube integrity in vitro, whether passage through a constricted environment ${ }^{56}$, like the filiform apparatus, contributes to the process remains to be determined. Increase in osmotic pressure has also been suggested as possibly underlying the species-specific ZmES4-induced pollen tube rupture that involves $\mathrm{K}^{+}$channel activation ${ }^{17,57}$. Whether ROS are involved in pre-conditioning the maize pollen tube for rupture is not known. Given that FER is conserved, ROS are ubiquitous, and pollen tubes from tobacco and lily also ruptured in response to ROS (Supplementary Fig. 6h,i), the FER-regulated pathway could be widespread to ensure reproductive success.

It is intriguing to consider how an elongating pollen tube survives the high ROS environment at the filiform apparatus yet ruptures almost instantaneously upon penetrating the synergid. We suggest that $\mathrm{Ca}^{2+}$ status across the synergid membrane at the filiform apparatus might underpin this process (Fig. 8). $\mathrm{A} \mathrm{Ca}^{2+}$ -dependent pollen tube rupture process (Figs 5 and 6) is consistent with a filiform apparatus $\left[\mathrm{Ca}^{2+}\right]$ on the extracellular side of the synergid cell membrane being below the threshold needed to induce rupture, preserving the integrity of a penetrating pollen tube. On the other hand, the synergid cytoplasmic $\mathrm{Ca}^{2+}$ level could be high ${ }^{58-60}$ and arrival of a pollen tube has been shown to increase the synergid $\left[\mathrm{Ca}^{2+}\right]$ in $A$. thaliana ${ }^{60}$. This could generate a $\left[\mathrm{Ca}^{2+}\right]$ high enough to trigger bursting of the invading tube. It could be envisaged that ROS encountered on the extracellular side of the synergid cell membrane could have weakened the pollen tube wall ${ }^{54}$, 'priming' it for rupture. Encountering the synergid cytoplasm, the already ROS-challenged tube would respond instantaneously to ROS-induced $\mathrm{Ca}^{2+}$-channel opening and abrupt water influx, leading to rupture. RAC/ROPs are known to regulate $\mathrm{Ca}^{2+}$ fluxes ${ }^{33,34}$, loss of FER could have obliterated the rise of $\left[\mathrm{Ca}^{2+}\right]$ in the fer female gametophyte, thus preventing the $\mathrm{Ca}^{2+}$-mediated rupture of the penetrating pollen tube. On the other hand, as pollen tubes engage the FER relatives ANX1 and ANX2 to maintain its integrity throughout the tube growth process prior to entering the synergid cell, it cannot be discounted that the pollen tube itself may also be autogenously involved in ensuring its own rupture once inside the synergid $^{27,28,61}$. Arrival at the female gametophyte and penetrating the synergid cell could have disengaged the function of ANX1 and ANX2, further lowering the ability of the penetrated pollen tube to resist rupture.

It has been shown that pollen tubes from Arabidopsis lyrata and more distantly related Cardamine flexuosa do not burst in A. thaliana female gametophyte ${ }^{24}$. Whether FER participates in controlling cross-species incompatibility has not yet been investigated. It is possible to speculate that these cross-species pollen tubes might not be able to trigger a crucial event, for example, a $\mathrm{Ca}^{2+}$ rise in the A. thaliana female gametophyte, as same-species pollen tubes do ${ }^{60}$, thus precluding a $\mathrm{Ca}^{2}+$ level high enough to mediate ROS-induced bursting. The recent demonstration that a MYB-factor-controlled transcriptional program is important for sperm release in A. thaliana indicates certain pollen tube quality is necessary in this female-male interplay ${ }^{19,21}$. Differences in these qualities on the A. lyrata tube surface might preclude its bursting in the A. thaliana female gametophyte. It will be interesting to explore in the future whether FER directly contributes to the observed barrier of A. thaliana fertilization by incompatible cross-species pollen tubes. 
a

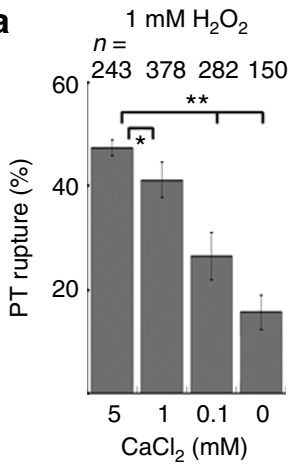

b

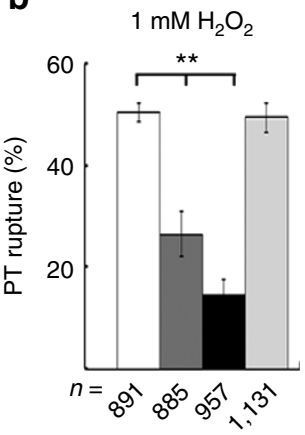

$200 \mu \mathrm{M} \mathrm{FeSO}_{4}$

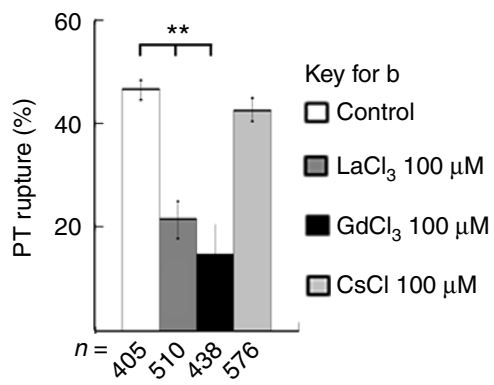

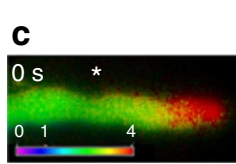

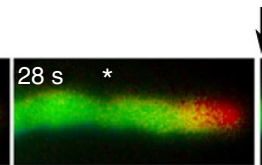

$\downarrow \mathrm{FeSO}_{4}$
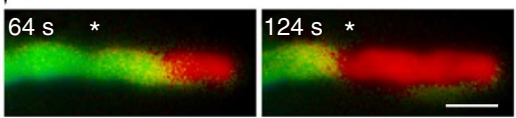

Burst
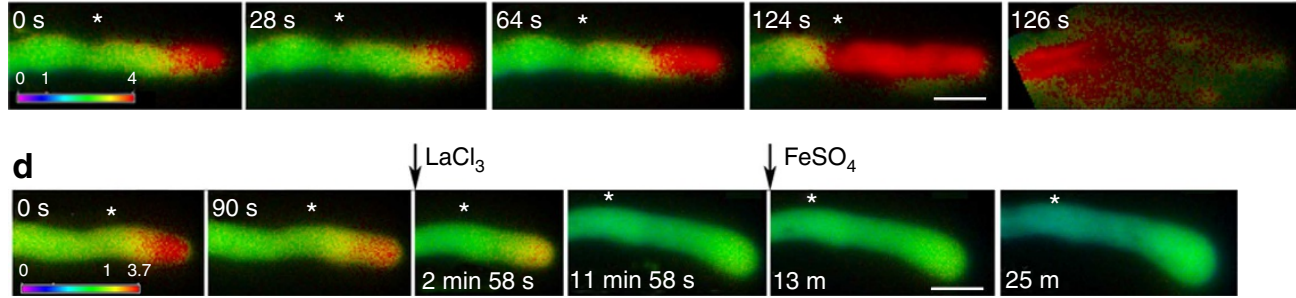

Figure 5 | ROS-induced pollen tube rupture is $\mathbf{C a}^{2+}$ dependent. (a) Effect of reducing $\left[\mathrm{Ca}^{2+}\right]$ on $\mathrm{H}_{2} \mathrm{O}_{2}$-induced pollen tube rupture. The excised pistils were pollinated and initially maintained in solidified medium containing $100 \mu \mathrm{M} \mathrm{Ca}^{2+}$ to allow germination and tube growth prior to application of liquid medium containing $5 \mathrm{mM}$ (standard) to $0 \mathrm{Ca}^{2+}$. The level of tube rupture under $0 \mathrm{Ca}^{2+}$ treatment condition reflected the presence of minuscule amount of residual $\mathrm{Ca}^{2}+$ contributed from the original growth medium. (b) Effects of inhibitors of $\mathrm{Ca}^{2}+$ channel $\left(\mathrm{LaCl}_{3}, \mathrm{GdCl}_{3}\right)$ and K $\mathrm{K}^{+}$channel $(\mathrm{CsCl})$ on ROS-induced rupture. Pollen tube rupture was suppressed by $\mathrm{LaCl}_{3}$ and $\mathrm{GdCl}_{3}$ in a dose-dependent manner but was insensitive to $\mathrm{CsCl}$ and control reagents $\mathrm{MgCl}_{2}$ and $\mathrm{BaCl}_{2}{ }^{17}$ (Supplementary Fig. 7a-d). (c,d) Imaging intracellular $\mathrm{Ca}^{2+}$ in a $\mathrm{Ca}^{2+}$-sensitive cameleon-expressing pollen tube during ROS $\left(250 \mu \mathrm{M} \mathrm{FeSO}_{4}\right)$-induced rupture (c) and in the presence of $100 \mu \mathrm{M} \mathrm{LaCl}_{3}$ (d). Heat maps reflect relative $\mathrm{Ca}^{2+}$ levels. The $0 \mathrm{~s}$ images show high $\mathrm{Ca}^{2+}$ at the tube apical region, typical of elongating pollen tubes ${ }^{1,42}$. ${ }^{*}$ indicate the same position on the pollen tubes as reference points for growth during the observation period. $\mathrm{LaCl}_{3}$-treated tube continued growth during the treatment period (see distance between * and tip), slowed and developed apical swelling (d), but did not burst. Supplementary Movies 3 and 4 show the time series in (c). Scale bar, $10 \mu \mathrm{m}$. Data $(\mathbf{a}, \mathbf{b})=$ average \pm s.e.m. from at least triplicate samples representative of three independent experiments; $n=$ total number of pollen tubes examined. ${ }^{\star \star} P \leq 0.01$ by Student's $t$-tests.
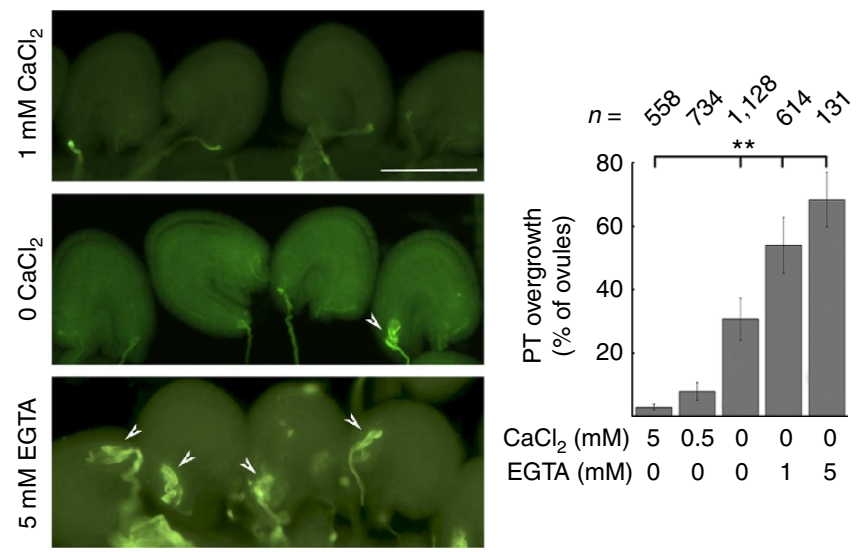

Figure 6 | $\mathrm{Ca}^{2}+$-dependent pollen tube rupture in the female gametophyte. Aniline blue-stained ovules show increasing level of pollen tube overgrowth in pollinated pistils fed with medium containing declining $\left[\mathrm{Ca}^{2+}\right]$ or added EGTA (data = average \pm s.e.m. from triplicate samples (each with at least three pistils) representative of three independent examinations for individual treatment conditions; $n=$ total number of ovules examined. ${ }^{\star \star} P \leq 0.01$ by Student's $t$-tests. Scale bar, $100 \mu \mathrm{m}$.

Understanding of how FER achieves its multiple functional roles is only emerging (Fig. 8). The fact that FER also controls ROS-mediated polarized root hair growth ${ }^{25}$ suggests these universal signalling molecules ${ }^{39,47,48}$ could be common links for how FER mediates some of its diverse biological roles. The ROPGEF-RAC/ROP molecular switch serving as signal mediators for FER provides a perfect nexus where multiple signals can be integrated and diverse responses can be dispatched through a battery of distinct effectors ${ }^{36,37}$ (Fig. 8). Incorporating additional molecules, like LRE which functions almost as prominently as FER itself ${ }^{14,15}$, to mediate a specific response, in this case ROSinduced pollen tube rupture (Fig. 7; Supplementary Fig. 8), might be a strategy whereby different FER functions can be executed depending on the presence of distinct partners. That the FERcontrolled dual function in pollen tube-ovule interaction can be uncoupled (Fig. 3c) is also indicative of FER having the capacity to utilize distinct mechanisms to mediate different functions, even ones that are intimately linked.

\section{Methods}

Plant growth conditions. Arabidopsis thaliana was maintained at $22^{\circ} \mathrm{C}$ in growth chambers ${ }^{25}$. Wild-type Col-0 and C24 were used as controls for fer-4 and srn, respectively. fer plants had pleiotropic phenotypes ${ }^{12,13,25,29-31}$, and were noticeably smaller and more stressed than wild type. Only the most robust fer plants were used in the analysis of their reproductive phenotype. Ire-5 was as described ${ }^{15}$ pHTR10::HTR10-RFP-transformed Arabidopsis ${ }^{62}$ was used as a marker line for sperm cells. Lat52:dtTomato transformed Arabidopsis was a gift from B. McClure (U. Missouri); it provided red fluorescence-labelled pollen tubes. Tobacco and lily were grown in greenhouses.

Pollen tube growth assays. Natural or hand-pollinated Arabidopsis pistils were used for in planta pollen tube growth analysis. The semi-in vivo pollen tube growth system $^{4,42}$ was used for in vitro tube growth analysis. A pistil-feeding system was developed to manipulate pistillate conditions and analysis of pollen tube growth in ovules. Each is described in the following sections. 

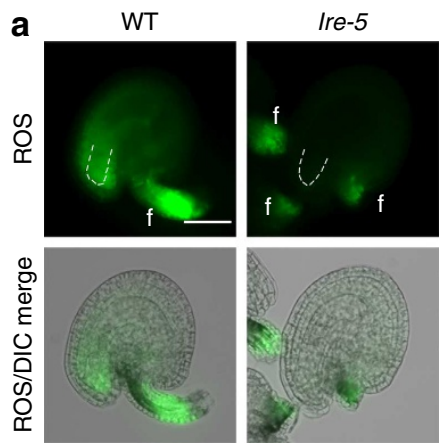

b

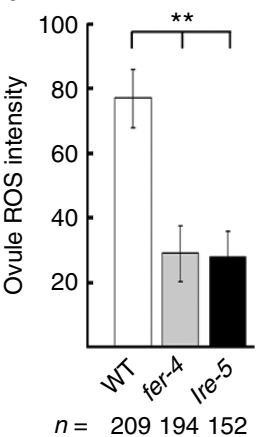

C

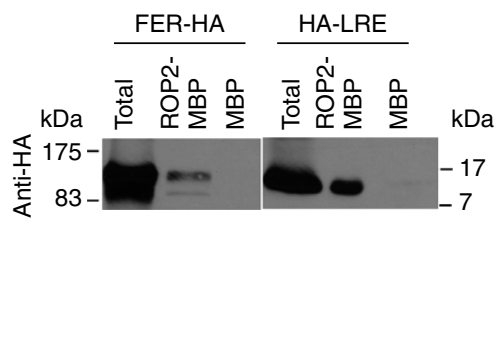

Figure 7 | LRE contributes to female gametophytic ROS and is a component of the FER-RAC/ROP signaling complex. (a) $\mathrm{H}_{2} \mathrm{DCF}-\mathrm{DA}$-stained wild-type and Ire-5 ovular ROS. f, funiculus, ROS occurred randomly, probably due to wound at the excision. (b) Quantification of filiform apparatus/synergid cell ROS in wild-type and Ire-5 ovules. Supplementary Fig. 8 shows comparable levels of fer-4 and Ire-5 (refs 14,15) female gametophytes displayed pollen tube overgrowth. Quantitative data are averages \pm s.e.m. of triplicate samplings (each with at least five pistils) from an analysis representative of three independent experiments; ${ }^{\star \star}$, significant differences, $P<0.01$ by Student's $t$-test. $n=$ total number of ovules examined. (c) ROP2-MBP pull down of FER-HA and HA-LRE. Immunoblot (upper) was stained by anti-HA antibodies to show the pulled down proteins. The FER pull-down result was similar to previously reported $^{25}$ and provides a comparison for LRE interaction with ROP2-MBP. The original scan and Ponceau-stained blot are shown in Supplementary Fig. 9.

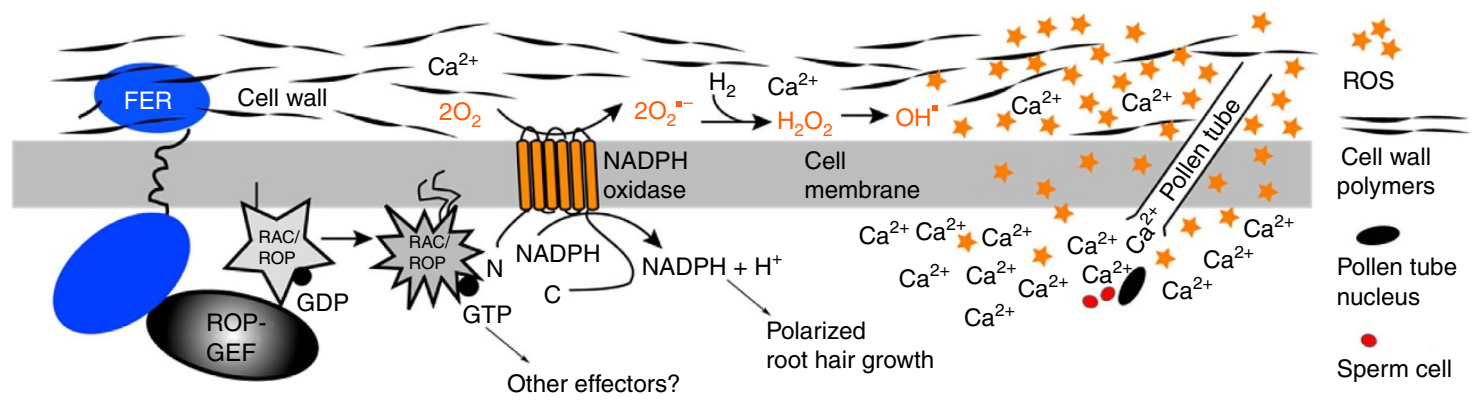

Figure 8 | RAC/ROPs and ROS as mediators of FER signaling. Both RAC/ROPs and ROS are known to regulate myriad biological processes, thus ideal for mediating the diverse functional roles of FER. NADPH oxidase is thus far the only downstream effector ${ }^{70}$ demonstrated to mediate two major FER functional roles, polarized root hair growth and pollen tube rupture in the female gametophyte.

In planta analysis of pollen-ovule interaction. Natural or hand-pollinated pistils from developmental stages $12-16$ flowers $^{40}$ were used. Ovules in stage 14 and 15 flowers were at peak ovule receptivity ${ }^{40,41}$ (Supplementary Fig. 4a). By stage 16, fertilization had occurred, sepals and petals had withered. Under our growth conditions, stages $13-15$ spanned $36 \mathrm{~h}$. For natural pollination, ovules from stages 14-15 flowers were used. For hand-pollination, stage $12 \mathrm{~b}$ flowers based on female gametophyte development ${ }^{41}$ (refer to as stage 12 in the text for simplicity) were emasculated and pollinated $\sim 17 \mathrm{~h}$ later at the onset of stage $13.20 \mathrm{~h}$ after pollination, pistils were fixed in FAA (10\% formaldehyde, $5 \%$ acetic acid, $50 \%$ ethyl alcohol), softened in $1 \mathrm{M} \mathrm{NaOH}$ for $4 \mathrm{~h}$, and stained with aniline blue to visualize pollen tubes ${ }^{63}$. Wild-type and pollen-specific Lat52::tdTomato ${ }^{64}$ transformed pollen were used. Aniline blue-stained pollen tubes were visualized by epifluorescence (EX330-380/DM 400/BA420) in pistil squashes. tdTomato fluorescence protein-labelled pollen tubes were observed by epifluorescence (Ex546/10/DM 565 LP/Em 590 LP) after ovule excision.

Analysis of pollen tube rupture in vitro. The semi-in vivo pollen tube growth system $^{4,42}$ was used for in vitro Arabidopsis pollen tube growth and rupture studies because it supports robust in vitro growing pollen tubes and low levels of spontaneous rupture (see Fig. 4a,c and Supplementary Fig. 7e,f, see the standard cultures with $10 \%$ sucrose samples). Stage $12 \mathrm{~b}$ flowers were emasculated and pollinated $\sim 17 \mathrm{~h}$ later. Pollinated pistils were excised at the style/ovary junction and maintained in $3.5 \mathrm{~cm}$ petri dishes on solidified ( $0.7 \%$ agarose) germination medium [GM] [5 $\mathrm{mM} \mathrm{CaCl}_{2}, 5 \mathrm{mM} \mathrm{KCl}, 1.6 \mathrm{mM} \mathrm{HBO}_{3}, 10 \%$ sucrose, $\left.\mathrm{pH} 7.5\right]^{65}$, unless modified as indicated in the text or figure legends. Pollen tubes emerged from the cut pistil after $\sim 2-3 \mathrm{~h}$. For mock and ROS treatments, standard medium or medium supplemented with various reagents, respectively were added around $1.5-2 \mathrm{~h}$ after pollen tubes had emerged from the cut pistils and observations were made $1 \mathrm{~h}$ after application of reagents except otherwise indicated. For treatments, all reagents were dissolved as suggested by manufacturers and diluted into $1 \mathrm{ml}$ standard or modified GM for application to pollen tube culture. Tobacco and lily in vitro pollen tube growth conditions were as described ${ }^{66}$. Tobacco pollen tubes were cultured in $0.8 \mu \mathrm{M} \mathrm{MgSO} \mathrm{Mg} \mathrm{H}_{2} \mathrm{O}, 1.6 \mu \mathrm{M} \mathrm{H}_{3} \mathrm{BO}_{3}, 3 \mathrm{mM} \mathrm{Ca}\left(\mathrm{NO}_{3}\right)_{2} .4 \mathrm{H}_{2} \mathrm{O}$, $1 \mathrm{mM} \mathrm{KNO}_{3}, 10 \mathrm{mM}$ MES, pH $6.0,8 \%$ sucrose, solidified with $0.7 \%$ agarose. Lily pollen tubes were cultured in $1.6 \mu \mathrm{M} \mathrm{H}_{3} \mathrm{BO}_{3}, 1 \mathrm{mM} \mathrm{KCl}, 0.1 \mathrm{mM} \mathrm{CaCl}, 15 \mathrm{mM}$ MES, pH 5.5, 7\% sucrose, solidified with $0.7 \%$ agarose.

For ROS treatments: three different ROS reagents were used. First, $\mathrm{H}_{2} \mathrm{O}_{2}$ (Fisher) was added to pollen tube cultures directly at the concentrations indicated. Second, $100 \mu \mathrm{M}$ xanthine (Alfa Aesar)/0.2 U [X/XO] (CalBiochem) was added to generate superoxide. Third, $\mathrm{FeSO}_{4}$ (Fisher) at the indicated concentration made in GM (adjusted to $\mathrm{pH} 6$ to optimize the Fenton reaction without noticeably affecting pollen tube growth in control mock treatments) was added to pollen tube cultures to generate hydroxyl radicals via the Fenton reaction ${ }^{43,44,67}$.

For ROS scavenger treatments: the hydroxyl radical scavengers sodium benzoate (Acros Organics), adenine (Sigma) and sodium salicylate (Sigma) and $\mathrm{H}_{2} \mathrm{O}_{2}$ scavenger $\mathrm{KI}$ or sodium pyruvate ${ }^{38,67}$ were applied in $1 \mathrm{ml}$ of standard GM or modified with various reagents at the concentrations indicated in the figures for 10 min before application of $1 \mathrm{mM} \mathrm{H}_{2} \mathrm{O}_{2}$ or $200 \mu \mathrm{M} \mathrm{FeSO}_{4}$.

To determine the effect of $\mathrm{Ca}^{2+}$ on ROS-induced pollen tube bursting, $1 \mathrm{ml}$ of modified GM with $100 \mu \mathrm{M} \mathrm{Ca}^{2+}$ was used in the solidified medium prior to ROS treatments. The low $\left[\mathrm{Ca}^{2+}\right]$ was needed to support pollen tube growth, which would not occur in $\mathrm{Ca}^{2+}$-free medium. For ROS treatment, $\mathrm{H}_{2} \mathrm{O}_{2}(1 \mathrm{mM}$, or otherwise indicated) or $\mathrm{FeSO}_{4}(200 \mu \mathrm{M})$ was applied in $1 \mathrm{ml}$ of GM containing $0-5 \mathrm{mM}$ (standard) $\mathrm{Ca}^{2+}$. Transformed Arabidopsis pollen tubes expressing the $\left[\mathrm{Ca}^{2+}\right]$ reporter yellow Cameleon YC3.6 (ref. 46) were used to monitor intracellular $\left[\mathrm{Ca}^{2+}\right]$ during ROS treatment $\left(250 \mu \mathrm{M} \mathrm{FeSO}_{4}\right)$.

To examine the involvement of $\mathrm{Ca}^{2+}$ and $\mathrm{K}^{+}$channels in ROS-induced pollen tube bursting, $\mathrm{Ca}^{2+}$ channel inhibitors $\mathrm{GdCl}_{3}$ (MP Biomedicals, LLC) and $\mathrm{LaCl}_{3}$ (ref. 43) (Santa Cruz Biotechnologies), and $\mathrm{K}^{+}$channel inhibitor $\mathrm{CsCl}^{68}$ were added to pollen tube cultures $10 \mathrm{~min}$ prior to application of $\mathrm{H}_{2} \mathrm{O}_{2}(1 \mathrm{mM})$ or $\mathrm{FeSO}_{4}$ $(250 \mu \mathrm{M})$ in GM.

To examine the importance of osmoregulation for ROS-induced pollen tube bursting, $1 \mathrm{mM} \mathrm{H}_{2} \mathrm{O}_{2}$ was applied in GM containing amounts of sucrose (5-15\%) below or above, respectively, than in standard GM (10\%). 
Excised pistil-feeding assays. A pistil-feeding assay, whereby the pedicel of an excised pollinated pistil was immersed into medium (Fig. 3a) unsupplemented or supplemented with reagents, was developed to manipulate ovular conditions so as to examine factors that are important for pollen tube rupture. Pollen germination and tube growth in wild-type (Fig. 3a; Supplementary Fig. 5a) and fer-4 (Supplementary Fig. 5b) pistils were not noticeably affected under mock treatment conditions. The outer three whorls of stage $12 \mathrm{c}$ flowers ${ }^{40,41}$ were removed and their unpollinated pistils were excised from the base of the pedicel. The pedicel was planted into solidified $(0.7 \%$ agarose) standard or modified GM in a sealed pipet tip. Chemicals used included DPI, an inhibitor of flavin-containing enzymes commonly used to determine NADPH oxidase dependence of ROS production, the superoxide scavengers tiron and $\mathrm{CuCl}_{2}$, the $\mathrm{H}_{2} \mathrm{O}_{2}$ scavenger potassium iodide and sodium pyruvate and the hydroxyl radical scavenger sodium benzoate ${ }^{38,39,67}$ (Supplementary Fig. 3). Pistils were maintained for $6-8 \mathrm{~h}$ to allow reagent uptake, then pollinated by wild-type pollen, and kept for another $20 \mathrm{~h}$ in the same medium before processing for ovular ROS detection and aniline blue staining to visualize pollen tubes.

The pistil-feeding assay was modified to assess $\mathrm{Ca}^{2+}$ requirement on pollen tube rupture in the ovule because altering pistillate $\mathrm{Ca}^{2+}$ conditions prior to pollination inhibited pollen germination and tube growth. Pistils were hand pollinated in planta to allow pollen germination and tube growth into the stigmatic papillae for $90 \mathrm{~min}$ prior to pistil excision and placed on modified GM with declining $\mathrm{Ca}^{2+}$ or added EGTA. The excised pistils were laid sideways onto solidified GM (Supplementary Fig. 7g) for $20 \mathrm{~h}$ and then processed for observation. This allowed pistillate conditions to be altered without affecting pollen tube growth and targeting in the central transmitting tissues.

Detection of ovular ROS. Ovules were dissected from the pistil and immediately transferred to staining solutions at room temperature for whole mount observations. Staining and washes were carried out by absorbing used reagents by filter wedges and replenishing with new reagents or wash buffers. Each pistil was processed individually and routinely yielded $\sim 20-30$ undamaged ovules. $\mathrm{H}_{2} \mathrm{DCF}-\mathrm{DA}$ $\left[2^{\prime}, 7^{\prime}\right.$-dichlorodihydrofluorescein diacetate] (Sigma), is highly sensitive to hydroxyl radicals among other oxygenated species ${ }^{38,67}$; HPF (hydroxyphenyl fluorescein) (2-[6-(4'-hydroxy) phenoxy-3 H-xanthene-3-on-9-yl] benzoic acid (Cayman) is specific for hydroxyl radicals $\mathrm{s}^{38,67,69}$. Ovules were immersed in $50 \mu \mathrm{M} \mathrm{H}_{2}$ DCF-DA or $50 \mu \mathrm{M} \mathrm{HPF}$ in $50 \mu \mathrm{M} \mathrm{CaCl}_{2}, 5 \mu \mathrm{M} \mathrm{KCl}, 10 \mathrm{mM} \mathrm{MES}, \mathrm{pH} 6.15$ at room temperature for $15 \mathrm{~min}$. Samples were washed and observed immediately within a 5-min period. When used, DPI and various ROS scavengers described above were added under ROS-staining condition to concentrations described in the text. Where not indicated, data were from $\mathrm{H}_{2}$ DCF-DA staining. We also tested Oxyburst green $\mathrm{H}_{2} \mathrm{HFF}-\mathrm{BSA}$ (Invitrogen) that detects extracellular superoxide. It did not show signals internal to the micropyle, precluding its use in our studies. Imaging was carried out on a NIKON Eclipse E800 and captured by a SPOT CCD camera. $\mathrm{H}_{2}$ DCF-DA- and HPF-detected ROS and JIM5-immunodetected pectin were observed by epifluorescence (Ex460-500, DM505, BA510-560).

Data analysis and quantitative data presentation. Unless otherwise indicated, data bars in histograms are the average \pm s.e.m. of at least three replicates from one representative experiment, which was repeated at least three times with comparable results. Experiments scoring ovule phenotypes examined at least three pistils (each of which yielded 20-30 undamaged ovules) per replicate. The average number of ovules and pollen tubes examined for the data shown are indicated in the figures as (n). Student's $t$-test was used for statistical analysis. Differences with $P \leq 0.05$ or $P \leq 0.01$ (designated respectively by ${ }^{*}$ and ${ }^{* *}$ in figures) are considered significant. In most experiments, differences between control and experimental samples had $P \ll 0.01$. Average signal intensity in regions of interest of identical areas at the filiform apparatus region (as shown in Supplementary Fig. 2) was quantified by ImageJ.

Protein pull-down assay. Escherichia coli produced ROP2-MBP and protoplastexpressed FER-HA and HA-LRE were used in protein pull-down assays as previously described ${ }^{25}$. Briefly, ROP2-MBP-bound amylose resin was used to pulldown FER-HA and HA-LRE expressed from the CaMV35S promoter in Arabidopsis protoplasts. $0.4 \%$ Triton X-100 soluble protoplast proteins were bound to the resin in $40 \mathrm{mM}$ Tris- $\mathrm{HCl}$ (pH 7.5), $100 \mathrm{mM} \mathrm{NaCl}, 1 \mathrm{mM} \mathrm{Na}_{2}$-EDTA. After binding, the resin was washed five times in the same buffer. The bound proteins were eluted by direct resuspension and boiling in SDS-PAGE loading buffer and used for immunoblot analysis. Protein blots were stained by Ponceau (Sigma) to ensure comparable amounts of bait proteins were used. Anti-HA antibodies (Santa Cruz) were used to detect the pulled down FER-HA and HA-LRE.

\section{References}

1. Cheung, A. Y. \& Wu, H.-M. Structural and signaling networks for the polar cell growth machinery in pollen tubes. Annu. Rev. Plant Biol. 59, 547-572 (2008).

2. Cheung, A. Y., Boavida, L., Aggarwal, M., Wu, H.-M. \& Feijo, J. The pollen tube journey in the pistil and imaging the in vivo process by two-photon microscopy. J. Exp. Bot. 61, 1907-1915 (2010).
3. Dresselhaus, T. \& Franklin-Tong, V. Male-female crosstalk during pollen germinatino, tube growth and guidance, and double fertilization. Mol. Plant 6, 1018-1036 (2013).

4. Cheung, A. Y., Wang, H. \& Wu, H.-M. A floral transmitting tissue-specific glycoprotein attracts pollen tubes and stimulates their growth. Cell 82, 383-393 (1995).

5. Wu, H.-M., Wang, H. \& Cheung, A. Y. A pollen tube growth stimulatory glycoprotein is deglycosylated by pollen tubes and displays a glycosylation gradient in the flower. Cell 82, 395-403 (1995).

6. Chae, K. \& Lord, E. M. Pollen tube growth and guidance: roles of small, secreted proteins. Ann. Bot. 108, 627-636 (2011).

7. Okuda, S. et al. Defensin-like polypeptide LUREs are pollen tube attractants secreted from synergid cells. Nature 458, 357-361 (2009).

8. Marton, M. L., fastner, A., Uebler, S. \& Dresselhaus, T. Overcoming hybridization barriers by the secretion of the maize pollen tube attractant ZmEA1 from Arabidopsis ovules. Curr. Biol. 22, 1194-1198 (2012).

9. Kasahara, R. D., Portereiko, M. F., Sandaklie-Nikolova, L., Rabiger, D. S. \& Drews, G. N. MYB98 is required for pollen tube guidance and synergid cell differentiation in Arabidopsis. Plant Cell 17, 2981-2992 (2005).

10. Leshem, Y., Johnson, C. \& Sundaresan, V. Pollen tube entry into the synergid cell of Arabidopsis is observed at a site distinct from the filiform apparatus. Plant Reprod. 16, 93099 (2013).

11. Palanivelu, R. \& Tsukamoto, T. Pathfinding in angiosperm reproduction: pollen tube guidance by pistils ensures successful double fertilization. WIREs Dev. Biol. 1, 96-113 (2012).

12. Rotman, N. et al. Female control of male gamete delivery during fertilization in Arabidopsis thaliana. Curr. Biol. 13, 432-436 (2003).

13. Huck, N., Moore, J. M., Federer, M. \& Grossniklaus, U. The Arabidopsis mutant feronia disrupts the female gametophytic control of pollen tube reception. Development 130, 2149-2159 (2003).

14. Capron, A. et al. Maternal control of male-gamete delivery in Arabidopsis involves a putative GPI-anchored protein encoded by the LORELEI gene. Plant Cell 20, 3038-3049 (2008).

15. Tsukamoto, T., Qin, Y., Huang, Y., Dunatunga, D. \& Palanivelu, R. A role for LORELEI, a putative glycosylphosphatidylinositol-anchored protein, in Arabidopsis thaliana double fertilization and early seed development. Plant $J$. 62, 571-588 (2010).

16. Kessler, S. A. et al. Conserved molecular components for pollen tube reception and fungal invasion. Science 330, 968-971 (2010).

17. Amien, S. et al. Defensin-like ZmES4 mediates pollen tube burst in maize via opening of the potassium channel KZM1. PLoS Biol. 8, e1000388 (2010).

18. Schiott, M. et al. A plant plasma membrane $\mathrm{Ca}^{2+}$ pump is required for normal pollen tube growth and fertilization. Proc. Natl Acad. Sci. USA 101, 9502-9507 (2004).

19. Leydon, A. R. et al. Three MYB transcription factors control pollen tube differentiation required for sperm release. Curr. Biol. 23, 1209-1214 (2013).

20. Kasahara, R. D. et al. Fertilization recovery after defective sperm cell release in Arabidopsis. Curr. Biol. 12, 1084-1089 (2012).

21. Liang, Y. et al. MYB97, MYB101, and MYB120 function as male factors that control pollen tube-synergid interaction in Arabidopsis thaliana fertilization. PLoS Genet. 11, e1003933 (2013).

22. Cheung, A. Y. \& Wu, H.-M. THESEUS1, FERONIA and relatives: a family of cell wall sensing receptor kinases? Curr. Opin. Plant Biol. 14, 632-641 (2011)

23. Hématy, K. et al. A receptor-like kinase mediates the response of Arabidopsis cells to the inhibition of cellulose synthesis. Curr. Biol. 17, 922-931 (2007).

24. Escobar-Restrepo, J.-M. et al. The FERONIA receptor-like kinase mediates male-female interactions during pollen tube reception. Science 317, 656-660 (2007).

25. Duan, Q., Kita, D., Li, C., Cheung, A. Y. \& Wu, H.-M. FERONIA receptor-like kinase regulates RHO GTPase signaling of root hair development. Proc. Natl Acad. Sci. USA 107, 17821-17826 (2010).

26. Zimmermann, P., Hirsch-Hoffmann, M., Hennig, L. \& Gruissem, W. GENEVESTIGATOR. Arabidopsis microarray database and analysis toolbox. Plant Physiol. 136, 2621-2632 (2004).

27. Miyazaki, S. et al. ANUXR1 and 2, sister genes to FERONIA/SIRENE, are male factors for coordinated fertilization. Curr. Biol. 19, 1327-1331 (2009).

28. Boisson-Dernier, A. et al. Disruption of the pollen-expressed FERONIA homologs ANXUR1 and ANXUR2 triggers pollen tube discharge. Development 136, 3279-3288 (2009).

29. Gou, H. et al. Three related receptor-like kinases are required for optimal cell elongation in Arabidopsis thaliana. Proc. Natl Acad. Sci. USA 106, 7648-7653 (2009).

30. Deslauriers, S. D. \& Larsen, P. B. FERONIA is a key modulator of brassinosteroid and ethylene responsiveness in Arabidopsis hypocotyls. Mol. Plant 3, 626-640 (2010).

31. Yu, F. et al. FERONIA receptor kinase pathway suppresses abscisic acid signaling in Arabidopsis by activating ABI2 phosphatase. Proc. Natl Acad. Sci. USA 109, 14693-14698 (2012). 
32. Berken, A., Thomas, C. \& Wittinghofer, A. A new family of RhoGEFs activates the Rop molecular switch in plants. Nature 436, 1176-1180 (2005).

33. Nibau, C., Wu, H.-M. \& Cheung, A. Y. RAC/ROP GTPases: 'Hubs' for signal integration and diversification in plants. Trends Plant Sci. 11, 310-315 (2006).

34. Yang, Z. \& Fu, Y. ROP/RAC GTPase signaling. Curr. Opin. Plant Biol. 10, 490-494 (2007)

35. Yalovsky, S., Bloch, D., Sorek, N. \& Kost, B. Regulation of membrane trafficking, cytoskeleton dynamics, and cell polarity by ROP/RAC GTPases. Plant Physiol. 147, 1527-1543 (2008).

36. Wu, G., Gu, Y., Li, S. \& Yang, Z. A genome-wide analysis of Arabidopsis Ropinteractive CRIB motif-containing proteins that act as Rop GTPase targets. Plant Cell 13, 2841-2856 (2001).

37. Lavy, M. et al. A Novel ROP/RAC effector links cell polarity, root-meristem maintenance, and vesicle trafficking. Curr. Biol. 17, 947-952 (2007).

38. Liszkay, A., van der Zalm, E. \& Schopfer, P. Production of reactive oxygen intermediates (superoxide, $\mathrm{H}_{2} \mathrm{O}_{2}$, and hydroxyl radical) by maize roots and their role in wall loosening and elongation growth. Plant Physiol. 136, 3114-3123 (2004)

39. Carol, R. \& Dolan, L. The role of reactive oxygen species in cell growth: lessons from root hairs. J. Exp. Bot. 57, 1829-1834 (2006).

40. Smyth, D. R., Bowman, J. L. \& Meyerowitz, E. M. Early flower development in Arabidopsis. Plant Cell 2, 755-767 (1990)

41. Christensen, C., A., King, E. J., Jordan, J. R. \& Drews, G. N. Megagametogenesis in Arabidopsis wild type and the Gf mutant. Sex Plant Reprod. 10, 49-64 (1997).

42. Palanivelu, R. \& Preuss, D. Distinct short-range ovule signals attract or repel Arabidopsis thaliana pollen tubes in vitro. BMC Plant Biol. 6, 7 (2006).

43. Demidchik, V., Shabala, S. N., Coutts, K. B., Tester, M. A. \& Davies, J. M. Free oxygen radicals regulate plasma membrane $\mathrm{Ca}^{2+}$ - and $\mathrm{K}^{+}$permeable channels in plant root cells. J. Cell Sci. 116, 81-88 (2003).

44. Foreman, J. et al. Reactive oxygen species produced by NADPH oxidase regulate plant cell growth. Nature 422, 442-446 (2003).

45. Holdaway-Clarke, T. \& Hepler, P. K. Control of pollen tube growth: role of ion gradients and fluxes. New Phytol. 159, 539-563 (2003).

46. Iwano, M. et al. Fine-tuning of the cytoplasmic $\mathrm{Ca}^{2+}$ concentration is essential for pollen tube growth. Plant Physiol. 150, 1322-1334 (2009).

47. Swanson, S. \& Gilroy, S. ROS in plant development. Physiol. Plant 138, 384-392 (2010).

48. Jaspers, P. \& Kangasjarvi, J. Reactive oxygen species in abiotic stress signaling. Physiol. Plant 138, 405-413 (2010).

49. Cardenas, L., McKenna, S. T., Kunkel, J. G. \& Hepler, P. K. NAD(P)H oscillates in pollen tubes and is correlated with tip growth. Plant Physiol. 142, 1460-1468 (2006).

50. Potocký, M., Jones, M. A., Bezvoda, R., Smirnoff, N. \& Zarsky, V. Reactive oxygen species produced by NADPH oxidase are involved in pollen tube growth. New Phyto. 174, 742-751 (2007).

51. Wilkins, K. A. et al. Reactive oxygen species and nitric oxide mediate actin reorganization and programmed cell death in the self-incompatibility response of papaver. Plant Physiol. 156, 404-416 (2011).

52. Cosgrove, D. J. Growth of the plant cell wall. Nat. Rev. Mol. Cell Biol. 6, 850-861 (2005).

53. Carol, R. J. et al. A RhoGDP dissociation inhibitor spatially regulates growth in root hair cells. Nature 438, 1013-1016 (2005).

54. Fry, S. C., Dumville, J. C. \& Miller, J. G. Fingerprinting of polysaccharides attacked by hydroxyl radicals in vitro and in the cell walls of ripening pear fruit. Biochem. J. 357, 729-737 (2001).

55. Woriedh, M. et al. External application of gametophyte-specific ZmPMEI1 induces pollen tube burst in maize. Plant Reprod. 26, 255-266 (2013).

56. Nezhad, A. S., Naghavi, M., Packirisamy, M., Bhat, R. \& Geitmann, A Quantification of cellular penetrative forces using lab-on-a-chip technology and finite element modeling. Proc. Natl Acad. Sci. USA 110, 8093-8098.

57. Franklin-Tong, V. E. Plant fertilization: bursting pollen tubes! Curr. Biol. 20, R681-R683 (2010).

58. Tian, H.-Q. \& Russell, S. D. Calcium distribution in fertilized and unfertilized ovules and embryo sacs of Nicotiana tabacum L. Planta 202, 93-105 (1997).
59. Kristóf, Z., Tímár, O. \& Imre, K. Changes of calcium distribution in ouvles of Torenia fournieri during pollination and fertilization. Protoplasma 208, 149-155 (1990).

60. Iwano, M. et al. Cytoplasmic $\mathrm{Ca}^{2+}$ changes dynamically during the interaction of the pollen tube with synergid cells. Development 139, 4202-4209 (2012).

61. Nibau, C. \& Cheung, A. Y. The CrRLK family of receptor kinases- a novel and conserved role in the control of polar cell growth: a review. Plant Signal Behav. 6, 652-656 (2011).

62. Ingouff, M. et al. Distinct dynamics of HISTONES3 variants between the two fertilization products in plants. Curr. Biol. 17, 1032-1037 (2007).

63. Mori, T., Kuroiwa, H., Higashiyama, T. \& Kuroiwa, T. Generative cell specific 1 is essential for angiosperm fertilization. Nat. Cell Biol. 8, 64-71 (2006).

64. Shaner, N. C. et al. Improved monomeric red, orange and yellow fluorescent proteins derived from Discosoma sp. Red fluorescent protein. Nat. Biotech. 22, 1567-1572 (2004).

65. Boavida, L. C. \& McCormick, S. Temperature as a determinant factor for increased and reproducible in vitro pollen germination in Arabidopsis thaliana. Plant J. 52, 570-582 (2007).

66. Cheung, A. Y. et al. The dynamic pollen tube cytoskeleton: live cell studies using actin-binding and microtubule-binding reporter proteins. Mol. Plant 1, 686-702 (2008).

67. Dunand, C., Crevecoeur, M. \& Penel, C. Distribution of superoxide and hydrogen peroxide in Arabidopsis root and their influence on root development: possible interaction with peroxidases. New Phytol. 174, 332-341 (2006).

68. Becker, D. et al. Changes in voltage activation, $\mathrm{Cs}^{+}$sensitivity, and ion permeability in $\mathrm{H} 5$ mutants of the plant $\mathrm{K}^{+}$channel KAT1. Proc. Natl Acad. Sci. USA 93, 8123-8128 (1996).

69. Setsukinai, K. et al. Development of novel fluorescence probes that can reliably detect reactive oxygen species and distinguish specific species. J. Biol. Chem. 278, 3170-3175 (2003).

70. Wong, H. L. et al. Regulation of rice NADPH oxidase by binding of Rac GTPase to its N-terminal extension. Plant Cell 19, 4022-4034 (2007).

\section{Acknowledgements}

We thank F. Berger (National University of Singapore) for the srn seeds, Ravi Palanivelu (University of Arizona) for lre-5 seeds, M. Johnson (Brown U.) for pHTR10::HTR10-RFP seeds, B. McClure (U. of Missouri) for Lat52::tdTomato seeds, M. Iwano (Nara Institute of Science and Technology, Japan) for Lat52::cameleon 3.6 seeds and Y.-j. Zou for the whole pistil images shown in Fig. 3a and Supplementary Fig. 5a. We also thank Ravi Palanivelu and Mark Johnson for discussions. E.A.J. and L.G. were partially supported by Summer Undergraduate Research Fellowships from the ASPB, a Linda Slakey Award and a Torrey Scholarship from University of Massachusetts. This work was supported by the NSF (IOB-0544222, -1146941 and -1147165 to A.Y.C. and H.-M.W.).

\section{Author contributions}

H.-M.W. and A.Y.C. led the project conceptualization, design and manuscript preparation; Q.D. contributed to all experimental parts of the work; D.K. identified FER and ROPGEF interaction and contributed to mechanistic studies in ovules; M.A. did pilot in vitro pollen tube bursting experiment; E.A.J. did the majority of the in vitro pollen tube rupture studies; L.G. contributed the RFP-labelled sperm release study and carried out FER/LRE RAC/ROP interaction studies. All participated in manuscript writing.

\section{Additional information}

Supplementary Information accompanies this paper at http://www.nature.com/ naturecommunications

Competing financial interests: The authors declare no competing financial interests

Reprints and permission information is available online at http://www.npg.nature.com/ reprintsandpermissions/

How to cite this article: Duan, Q. et al. Reactive oxygen species mediate pollen tube rupture to release sperm for fertilization in Arabidopsis. Nat. Commun. 5:3129 doi: $10.1038 /$ ncomms4129 (2014) 